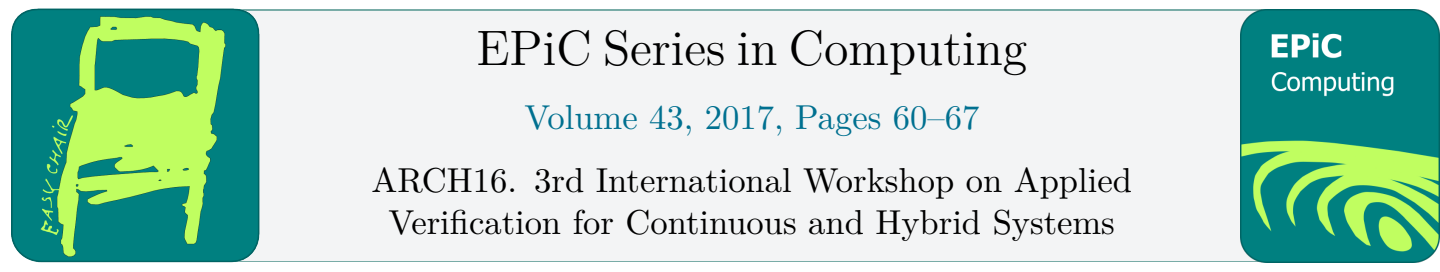

\title{
Large-Scale Linear Systems from Order-Reduction (Benchmark Proposal)
}

\author{
Hoang-Dung Tran, Luan Viet Nguyen, and Taylor T. Johnson \\ University of Texas at Arlington, Texas, USA \\ taylor.johnson@gmail.com
}

\begin{abstract}
This benchmark suite is composed of nine examples of large-scale linear systems, ranging in dimensionality in the tens to the low thousands. The benchmarks are derived from diverse fields such as civil engineering and robotics, and are based on similar existing test sets for model-order reduction algorithms in control and numerical analysis. Each example is provided in the SpaceEx XML model format as single-mode hybrid automaton and are compatible with the HyST model transformation tool to support analysis in other verification tools. Some preliminary reachability analysis results for some of the smaller examples (on the order of tens of dimensions) are presented using SpaceEx.

Category: academic Difficulty: low through challenge
\end{abstract}

\section{Context and Origins}

Symbolic state-space analysis has shown advantages in safety verification of continuous and hybrid systems in which the essential task is computing the set of reachable states symbolically with an iterative algorithm [1]. The main challenge of this approach is state-space explosion, which roughly is that the complexity of computation grows exponentially with the system dimensionality [2]. To implement efficiently symbolic reachability algorithms, significant effort has been invested in finding appropriate representations for the set of states that supports efficient operations used in the iterative computation. From classical polyhedral representations which are used in hybrid systems model checkers such as HyTech [3,4] and d/dt [5], more efficient representations such as zonotopes $[6-8]$ and support functions $[9,10]$ have been proposed and integrated in tools such as CORA and SpaceEx that use these state-of-the-art representations for analysis of hybrid systems with linear dynamics.

In spite of these advances, reachability analysis of large-scale systems with hundreds to thousands of dimensions is still infeasible even for linear time invariant (LTI) systems, i.e., without any discrete switching behavior. It is important to develop new techniques and tools that can be used to verify the safety of such high-dimensional systems, which usually exist in a broad range of fields and applications such as control systems, biological systems, analog circuits, and multi-agent systems.

To help test and evaluate reachability analysis methods and tools to enable verification of high-dimensional systems, we construct a set of benchmarks that are essentially LTI systems arising from model order reduction $[11,12]$. These benchmarks, which are models of practical 


\begin{tabular}{|l|l|l|l|l|l|}
\hline No. & Benchmark & Type & $\mathrm{n}$ & $\mathrm{m}$ & $\mathrm{p}$ \\
\hline 1 & Motor control system (MCS) & LTI & 8 & 2 & 2 \\
2 & Building model (BM) [12] & LTI & 48 & 1 & 1 \\
3 & International space station (ISS) [12] & LTI & 270 & 3 & 3 \\
4 & Partial differential equation (Pde) [12] & LTI & 84 & 1 & 1 \\
5 & FOM [12] & LTI & 1006 & 1 & 1 \\
6 & Modified nodal analysis model 1 [12](MNA-1) & LTI & 578 & 9 & 9 \\
7 & Modified nodal analysis model 5 [12](MNA-5) & LTI & 10913 & 9 & 9 \\
8 & Heat equation [12] & LTI & 200 & 1 & 1 \\
9 & Clamped beam model [12] & LTI & 348 & 1 & 1 \\
\hline
\end{tabular}

Table 2.1: Benchmarks for the order-reduction abstraction method in which $n$ is dimension of the system; $m$ and $p$ are the number of inputs and outputs respectively.

systems in different fields, have dimensions varying from ten to thousands. Each benchmark is given in the SpaceEx format as a single-mode hybrid automaton and can be easily transformed to other formats such as dReach [13] or Flow* [14] using the HyST model transformation tool [15]. Reachability analysis of some of the small and medium-size benchmarks (i.e., $<50$ dimensions ) are presented. These benchmarks may be effective to test and evaluate the scalability of verification approaches when dealing with large-size benchmarks (i.e. > 50 dimensions).

\section{Brief descriptions}

Since most of benchmarks are high-dimensional, their dynamic equations cannot be presented in detail in this paper. We refer readers to $[11,12]$ for for further details and derivations, as well as our provided supplementary material. ${ }^{1}$ The general form of the dynamics is:

$$
\begin{aligned}
\dot{x}(t) & =A x(t)+B u(t) \\
y(t) & =C x(t),
\end{aligned}
$$

where $x(t) \in \mathbb{R}^{n}$ is the system state, $y(t) \in \mathbb{R}^{p}$ is the system output, $u(t)$ is the control input, $A \in \mathbb{R}^{n \times n}, B \in \mathbb{R}^{n \times m}$, and $C \in \mathbb{R}^{p \times n}$.

In this section, we introduce briefly these benchmarks. Table 2.1 summarizes names, number of dimensions, and numbers of inputs and outputs of the benchmarks. The initial set of states, input constraints, and safety specifications of the benchmarks are given in Table 2.2.

Motor control system. The motor control system benchmark includes two motors that are controlled synchronously. Each motor has a local controller that is designed using pole placement method [16] to control the motor to satisfy: 1) the overshoot of the motor position is less than $16 \%$; 2) setting time is less than 0.04 seconds; 3) No steady-state error, even in the presence of a step disturbance input.

\footnotetext{
${ }^{1}$ The benchmarks are available online, http://verivital.com/hyst/benchmark-large-scale/
} 


\begin{tabular}{|c|c|c|c|}
\hline Benchmark & $\begin{array}{l}\text { Initial set of states } \\
X_{0}=\left\{x_{0} \in \mathbb{R}^{n} \mid l b(i) \leq x_{0}(i) \leq u b(i), 1 \leq i \leq n\right\}\end{array}$ & $\begin{array}{l}\text { Input constraint } \\
u=\left[u_{1}, \cdots, u_{m}\right]^{T}\end{array}$ & $\begin{array}{l}\text { Safety specification } \\
y=\left[y_{1}, \cdots, y_{p}\right]^{T}\end{array}$ \\
\hline $\begin{array}{l}\text { Motor con- } \\
\text { trol system }\end{array}$ & $\begin{array}{l}l b(i)=u b(i)=0, i=2,3,4,6,7,8 \\
l b(2)=0.002, u b(2)=0.0025 \\
l b(3)=0.001, u b(3)=0.0015\end{array}$ & $\begin{array}{l}u_{1} \in[0.16,0.3] \\
u_{2} \in[0.2,0.4]\end{array}$ & $\begin{array}{l}\text { unsafe region: } \\
0.35 \leq y_{1} \leq 0.4 \\
0.45 \leq y_{2} \leq 0.6\end{array}$ \\
\hline $\begin{array}{l}\text { Building } \\
\text { model }\end{array}$ & $\begin{array}{l}l b(i)=0.0002, u b(i)=0.00025,1 \leq i \leq 10 \\
l b(25)=-0.0001, u b(25)=0.0001 \\
l b(i)=u b(i)=0, \quad 11 \leq i \leq 48, i \neq 25\end{array}$ & $u_{1} \in[0.8,1]$ & $\begin{array}{l}\text { unsafe region: } \\
0.006<y_{1}\end{array}$ \\
\hline $\begin{array}{l}\text { Partial dif- } \\
\text { ferential } \\
\text { equation }\end{array}$ & $\begin{array}{l}l b(i)=0, u b(i)=0,1 \leq i \leq 64 \\
l b(i)=0.001, u b(i)=0.0015,64 \leq i \leq 80 \\
l b(i)=-0.002, u b(i)=-0.0015,81 \leq i \leq 84\end{array}$ & $u_{1} \in[0.5,1]$ & $\begin{array}{l}\text { safe region: } \\
y_{1} \leq 12\end{array}$ \\
\hline $\begin{array}{l}\text { International } \\
\text { space station }\end{array}$ & $l b(i)=-0.0001, u b(i)=0.0001,1 \leq i \leq 270$ & $\begin{array}{l}u_{1} \in[0,0.1] \\
u_{2} \in[0.8,1] \\
u_{3} \in[0.9,1]\end{array}$ & $\begin{array}{l}\text { Safe region: } \\
-0.0005 \leq y_{3} \leq 0.0005\end{array}$ \\
\hline FOM & $\begin{array}{l}l b(i)=-0.0001, u b(i)=0.0001,1 \leq i \leq 400 \\
l b(i)=0.0002, u b(i)=0.00025,401 \leq i \leq 800 \\
l b(i)=0, u b(i)=0,801 \leq i \leq 1006\end{array}$ & $u_{1} \in[-1,1]$ & $\begin{array}{l}\text { safe region: } \\
y_{1} \leq 45\end{array}$ \\
\hline MNA-1 & $\begin{array}{l}l b(i)=0.001, u b(i)=0.0015,1 \leq i \leq 2 \\
l b(i)=0, u b(i)=0,3 \leq i \leq 578\end{array}$ & $\begin{array}{l}u_{i}=0.1,1 \leq i \leq 5 \\
u_{i}=0.2,6 \leq i \leq 9\end{array}$ & $\begin{array}{l}\text { unsafe region: } \\
y_{1}>0.5\end{array}$ \\
\hline MNA- 5 & $\begin{array}{l}l b(i)=0.0002, u b(i)=0.00025,1 \leq i \leq 10 \\
l b(i)=0, u b(i)=0, \quad 11 \leq i \leq 10913\end{array}$ & $\begin{array}{l}u_{i}=0.1,1 \leq i \leq 5 \\
u_{i}=0.2,6 \leq i \leq 9\end{array}$ & $\begin{array}{l}\text { safe region: } \\
y_{1} \leq 0.2, y_{1} \leq 0.15\end{array}$ \\
\hline $\begin{array}{l}\text { Heat equa- } \\
\text { tion }\end{array}$ & $\begin{array}{l}l b(i)=0.6, u b(i)=0.625, \quad 1 \leq i \leq 2 \\
l b(i)=0, u b(i)=0,3 \leq i \leq 200\end{array}$ & $u_{1} \in[-0.5,0.5]$ & $\begin{array}{l}\text { safe region: } \\
y_{1} \leq 0.1\end{array}$ \\
\hline $\begin{array}{l}\text { Clamped } \\
\text { beam model }\end{array}$ & $\begin{array}{l}l b(i)=0, u b(i)=0,1 \leq i \leq 300 \\
l b(i)=0.0015, u b(i)=0.002,301 \leq i \leq 348\end{array}$ & $u_{1} \in[0.2,0.8]$ & $\begin{array}{l}\text { unsafe region: } \\
y_{1}>1000\end{array}$ \\
\hline
\end{tabular}

Table 2.2: Initial states, input constraints and safety specification for the outputs of the benchmarks.

Building model. The building model is a model of the Los Angeles University Hospital with 8 floors, each of which has 3 degrees of freedom [11]. This system has 48 state variables in which we are mostly interested in the twenty-fifth state $x_{25}(t)$, which is the motion of the first coordinate. The twenty-fifth state is the interested output of the building model and should not reach to the unsafe region given in Table 2.2.

Partial differential equation. The partial differential equation (PDE) is given by

$$
\frac{\partial x}{\partial t}=\frac{\partial^{2} x}{\partial z^{2}}+\frac{\partial^{2} x}{\partial v^{2}}+20 \frac{\partial x}{\partial v}-180 x+f(v, z) u(t)
$$

where $x$ is a function of time $t$, vertical position $v$ and horizontal position $z$. This problem lies on a square domain defined by two opposite points $(0,0)$ and $(1,1)$. The function $x(t, v, z)$ is zero on the boundaries of the square. A state-space equation of dimension of $N=n_{v} n_{z}$ of this $\mathrm{PDE}$ can be given by discretizing with centered difference approximation on a grid of $n_{v} \times n_{z}$ points. The input vector corresponding to $f(v, z)$ is composed of random elements while the output vector of the system is equated to the input vector for simplicity. The state-space model 
of PDE covered in this paper corresponds to the case of $n_{v}=7$ and $n_{z}=12$.

International Space Station (ISS). The ISS state-space model presented in this paper is a structural model of component 1R (Russian service module) of the International Space Station. It has 270 state variables with three inputs and three outputs.

FOM. This is state-space model of a dynamical system with following matrices:

$$
\begin{aligned}
& A=\left[\begin{array}{llll}
A_{1} & & & \\
& A_{2} & & \\
& & A_{3} & \\
& & & A_{4}
\end{array}\right], A_{1}=\left[\begin{array}{cc}
-1 & 100 \\
-100 & -1
\end{array}\right], A_{2}=\left[\begin{array}{cc}
-1 & 200 \\
-200 & -1
\end{array}\right], \\
& A_{3}=\left[\begin{array}{cc}
-1 & 400 \\
-400 & -1
\end{array}\right], A_{4}=\left[\begin{array}{cccc}
-1 & & & \\
& -2 & & \\
& & \ddots & \\
& & -1000
\end{array}\right] \\
& B^{T}=C=[\underbrace{10 \cdots 10}_{6} \underbrace{1 \cdots 1}_{1000}] \text {. }
\end{aligned}
$$

Modified nodal analysis model. The following Modified Nodal Analysis (MNA) equation is constituted from connecting voltage sources to the ports of a multiport:

$$
\begin{aligned}
E \dot{x}_{n} & =A x_{n}+B u_{p}, \\
i_{p} & =C x_{n},
\end{aligned}
$$

in which $i_{p}$ and $u_{p}$ are the port currents and voltages vectors respectively and

$$
A=\left[\begin{array}{cc}
-N & -G \\
G^{T} & 0
\end{array}\right], E=\left[\begin{array}{cc}
L & 0 \\
0 & H
\end{array}\right], x_{n}=\left[\begin{array}{l}
v \\
i
\end{array}\right],
$$

where $v$ and $i$ are variables of the MNA including node votages, inductor and voltage source currents, respectively. The matrices $-A$ and $E$ represent the conductance and susceptance matrices. The matrices $-N, L$ and $H$ contains the stamps for resistors, capacitors and inductors, respectively. Matrix $G$ consists of $1,-1$ and 0 , which describe the current variables in Kirchhoff's Current Law (KCL) equation. The input matrix $B$ and output matrix $C$ satisfy $B=C^{T}$. We give two MNA models with different number of state variables in the paper.

Heat equation. The state-space model of Heat equation is giving by discretizing the following equation:

$$
\left\{\begin{array}{l}
P D E \quad \frac{\partial}{\partial t} T(x, t)=\alpha \frac{\partial^{2}}{\partial x^{2}} T(x, t)+u(x, t), x \in(0,1) ; t>0, \\
B C s \quad T(0, t)=0=T(1, t), t>0, \\
I C \quad T(x, 0)=0, x \in(0,1) .
\end{array}\right\}
$$

where $T(x, t)$ represents the temperature field on a thin rod and $u(x, t)$ is the heat source. 


\begin{tabular}{|l|c|c|}
\hline \multirow{2}{*}{ Benchmark } & LGG & STC \\
\cline { 2 - 3 } & Time(s) & Time(s) \\
\hline Motor control system & 27 & N/A \\
\hline Building model & 893 & N/A \\
\hline Partial differential equation & OOT & N/A \\
\hline International space station & OOT & N/A \\
\hline FOM & OOT & N/A \\
\hline MNA-1 & OOT & N/A \\
\hline MNA-5 & OOT & N/A \\
\hline Heat equation & OOT & N/A \\
\hline Clamped beam model & OOT & N/A \\
\hline
\end{tabular}

Table 3.1: Computation cost for verification of the benchmarks using SpaceEx [10] with two scenarios LGG [19] and STC [20]. The terms of "N/A" and "OOT" mean "not applicable" and "out of time".

Clamped beam model. The state-space clamped beam model, which is obtained by spatial discretization of an appropriate partial different equation, has 348 states, one input and one output in which the input represents the force applied to the structure and the output is the displacement.

\section{Reachability analysis}

Since all benchmarks are LTI systems, there are different tools that can be used to analyze the safety of these benchmarks such as SpaceEx [10], CORA [17], CheckMake [18], DReach [13], and Flow* [14]. We specify each benchmark in the SpaceEx format as a single-mode hybrid automaton, which can be easily transformed to other formats using HyST [15].

Table 3.1 presents a preliminary overview of the computation cost of time-bounded reachability analysis for the benchmarks using SpaceEx. These experiments are conducted on a personal computer with the following configurations: Intel (R) Core(TM) i7-2677M CPU at $1.80 \mathrm{GHz}$, 4GB RAM, and 64-bit Window 7. The reachability analysis is conducted in a bounded time range $[0,20 s]$. The SpaceEx scenarios tested are LGG [19] and STC [20]. The sampling time is selected as 0.001 for all benchmarks. We note that the sampling time and time horizon should be selected appropriately based on the dynamics of specific system, for example, using the rule of thumb to pick the sampling time based on the inverse of the maximum eigenvalue. Intuitively, this would mean to pick large sampling times for slow dynamics and small sampling times for fast dynamics. Thus, while our preliminary results as shown in Table 3.1 indicate some examples are infeasible for analysis with SpaceEx, it is possible that a more careful selection of parameters would enable analysis of these systems, and we hope other researchers will be interested to try these examples. We set the upper limit for SpaceEx running time as two hours, and an experiment is said to be out of time (OOT) if we can not get the result after two hours. The reason the STC scenario did not produce results is due to the use of outputs as invariant conditions (i.e., $y=C x$ ) with nondeterministic dynamics, which does not seem to be supported when using STC.

Next, we present briefly the reachability analysis of some small and medium-size benchmarks (i.e., less than 50 dimensions). 


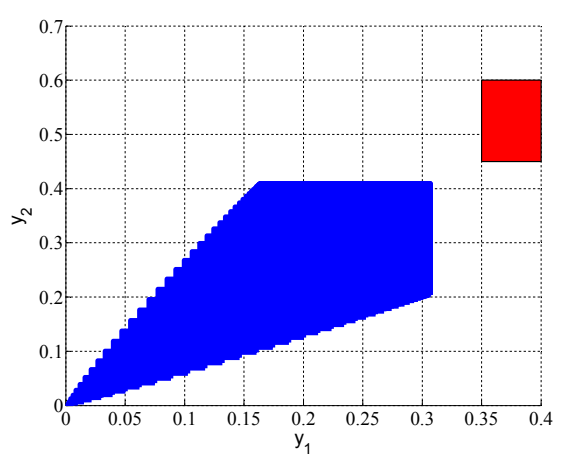

Figure 3.1: Reachable set of interested outputs of the motor control system (in $[0,20 s])$ and its corresponding unsafe region (the red region). The reachable set of interested outputs do not reach the unsafe region, thus the system is safe (in a bounded time interval $[0,20 s]$ ).

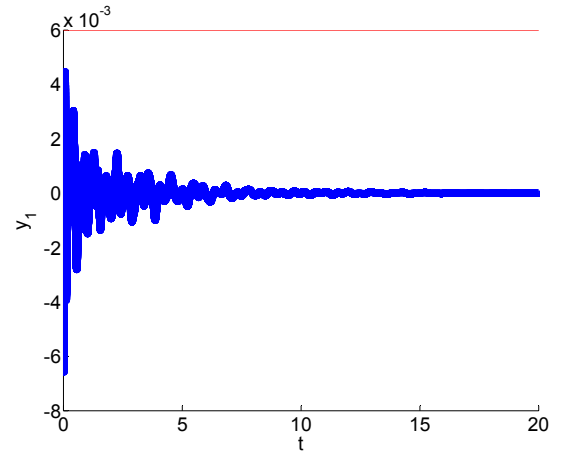

Figure 3.2: Reachable set of interested output of the building model system (in $[0,20 s])$ and its corresponding unsafe region (the region above the red line). The reachable set of interested output do not reach the unsafe region, thus the system is safe (in a bounded time interval $[0,20 s]$ ).

Motor control system. Figure 3.1 depicts the reachable set of the interested states of the motor control system. As shown in the figure, the reachable set does not reach to the unsafe region. Thus, we can conclude that the system is safe in the bounded time $[0,20 s]$. A stronger conclusion about the safety of the motor control system may be given by considering unbounded time safety verification.

Building model. Figure 3.2 depicts the reachable set of the this state of the building model. As can be seen from the figure, the reachable states of the output do not intersect the unsafe region. Thus, we can conclude that the system is safe in the bounded time [0,20s]. Similar to the above motor control system, a stronger conclusion about the safety of the building model may be given by considering unbounded time safety verification.

\section{Outlook}

Overall, we present in this paper a set benchmarks for purely continuous linear systems (i.e., LTI systems), modeled as single-mode hybrid automata in the SpaceEx model format. The benchmarks range in dimensionality from tens to thousands of dimensions, and come from many different domains. The continuous and hybrid verification community may use these benchmarks for comparing methods and tools, especially with respect to continuous post operator benchmarking for systems with a high number of dimensions. In ongoing and future work, we intend to introduce additional high-dimensional benchmarks with both piecewise affine dynamics and continuous dynamics including ones originally encoded as differential algebraic equations (DAEs), and are also investigating formalization of order-reduction methods as sound abstractions using approximate bisimulation relations [21]. 
Acknowledgements The material presented in this paper is based upon work supported by the National Science Foundation (NSF) under grant numbers CNS 1464311 and CCF 1527398, the Air Force Research Laboratory (AFRL) through contract number FA8750-15-1-0105, and the Air Force Office of Scientific Research (AFOSR) under contract number FA9550-15-1-0258. The U.S. government is authorized to reproduce and distribute reprints for Governmental purposes notwithstanding any copyright notation thereon. Any opinions, findings, and conclusions or recommendations expressed in this publication are those of the authors and do not necessarily reflect the views of AFRL, AFOSR, or NSF.

\section{References}

[1] P. Tabuada, Verification and control of hybrid systems: a symbolic approach. Springer Science \& Business Media, 2009.

[2] R. Pelánek, "Fighting state space explosion: Review and evaluation," in Formal Methods for Industrial Critical Systems. Springer, 2008, pp. 37-52.

[3] R. Alur, T. A. Henzinger, and P.-H. Ho, "Automatic symbolic verification of embedded systems," Software Engineering, IEEE Transactions on, vol. 22, no. 3, pp. 181-201, 1996.

[4] T. A. Henzinger, P.-H. Ho, and H. Wong-Toi, "Hytech: A model checker for hybrid systems," in Computer aided verification. Springer, 1997, pp. 460-463.

[5] E. Asarin, O. Bournez, T. Dang, and O. Maler, "Approximate reachability analysis of piecewiselinear dynamical systems," in Hybrid Systems: Computation and Control. Springer, 2000, pp. $20-31$.

[6] A. Girard, "Reachability of uncertain linear systems using zonotopes," in Hybrid Systems: Computation and Control. Springer, 2005, pp. 291-305.

[7] A. Girard, C. Le Guernic, and O. Maler, "Efficient computation of reachable sets of linear timeinvariant systems with inputs," in Hybrid Systems: Computation and Control. Springer, 2006, pp. 257-271.

[8] M. Althoff, O. Stursberg, and M. Buss, "Computing reachable sets of hybrid systems using a combination of zonotopes and polytopes," Nonlinear Analysis: Hybrid Systems, vol. 4, no. 2, pp. 233-249, 2010.

[9] C. Le Guernic and A. Girard, "Reachability analysis of hybrid systems using support functions," in Computer Aided Verification. Springer, 2009, pp. 540-554.

[10] G. Frehse, C. Le Guernic, A. Donzé, S. Cotton, R. Ray, O. Lebeltel, R. Ripado, A. Girard, T. Dang, and O. Maler, "Spaceex: Scalable verification of hybrid systems," in Computer Aided Verification. Springer, 2011, pp. 379-395.

[11] A. C. Antoulas, D. C. Sorensen, and S. Gugercin, "A survey of model reduction methods for large-scale systems," Contemporary Mathematics, vol. 280, pp. 193-219, 2001.

[12] Y. Chahlaoui and P. Van Dooren, "A collection of benchmark examples for model reduction of linear time invariant dynamical systems." 2002.

[13] S. Kong, S. Gao, W. Chen, and E. Clarke, "dreach: $\delta$-reachability analysis for hybrid systems," pp. 200-205, 2015.

[14] X. Chen, E. Ábrahám, and S. Sankaranarayanan, "Flow*: An analyzer for non-linear hybrid systems," in Computer Aided Verification. Springer, 2013, pp. 258-263.

[15] S. Bak, S. Bogomolov, and T. T. Johnson, "Hyst: a source transformation and translation tool for hybrid automaton models," in Proceedings of the 18th International Conference on Hybrid Systems: Computation and Control. ACM, 2015, pp. 128-133.

[16] R. C. Dorf and R. H. Bishop, "Modern control systems," 1998.

[17] M. Althoff, "An introduction to cora 2015," in Proc. of the Workshop on Applied Verification for Continuous and Hybrid Systems, 2015. 
[18] B. I. Silva, K. Richeson, B. Krogh, and A. Chutinan, "Modeling and verifying hybrid dynamic systems using checkmate," in Proceedings of 4 th International Conference on Automation of Mixed Processes, vol. 4. Citeseer, 2000, pp. 1-7.

[19] C. Le Guernic and A. Girard, "Reachability analysis of linear systems using support functions," Nonlinear Analysis: Hybrid Systems, vol. 4, no. 2, pp. 250-262, 2010.

[20] G. Frehse, R. Kateja, and C. Le Guernic, "Flowpipe approximation and clustering in space-time," in Proceedings of the 16th international conference on Hybrid systems: computation and control. ACM, 2013, pp. 203-212.

[21] H.-D. Tran, L. V. Nguyen, W. Xiang, and T. T. Johnson, "Order-reduction abstractions for safety verification of high-dimensional linear systems," arXiv, 2016. 